BULLETIN Bulletin hispanique

HISPANIQUE Université Michel de Montaigne Bordeaux

117-2 | 2015

Métamorphose(s) : représentations et réécritures

\title{
Écrire la métamorphose dans la littérature du Siècle d'Or
}

Philippe Rabaté

\section{(2) OpenEdition}

Journals

\section{Édition électronique}

URL : http://journals.openedition.org/bulletinhispanique/3960

DOI : 10.4000/bulletinhispanique.3960

ISSN : 1775-3821

Éditeur

Presses universitaires de Bordeaux

\section{Édition imprimée}

Date de publication : 15 décembre 2015

Pagination : 433-454

ISBN : 979-10-300-0041-2

ISSN : 0007-4640

Référence électronique

Philippe Rabaté, «Écrire la métamorphose dans la littérature du Siècle d'Or », Bulletin hispanique [En ligne], 117-2 | 2015, mis en ligne le 15 décembre 2018, consulté le 01 mai 2019. URL : http:// journals.openedition.org/bulletinhispanique/3960; DOI : 10.4000/bulletinhispanique.3960 


\title{
Écrire la métamorphose dans la littérature du Siècle d'Or
}

\author{
Philippe Rabaté \\ Université Paris-Ouest Nanterre La Défense
}

La présente étude procède à un état des lieux de la recherche sur la thématique des métamorphoses, en la mettant en relation avec l'idée de représentation et de monstre. Elle s'attache à étudier le lien qui se noue entre évocation de la métamorphose et poétique avant d'offrir quelques exemples littéraires de transformations qui constituent des variations sur le corps et ses états.

Mots-clés : métamorphoses, monstruosités, poétique, corps.

El presente estudio ofrece un estado de la cuestión sobre la investigación en torno a la temática de las metamorfosis, poniéndola en relación con la idea de representación y de monstruo. Procura estudiar el vínculo que se esboza entre evocación de la metamorfosis y poética de la obra antes de brindarle al lector unos ejemplos literarios de transformaciones que ofrecen variaciones sobre el cuerpo y sus distintos estados.

Palabras claves: metamorfosis, monstruosidad, poética, cuerpo.

This study sums up first the results of current research on metamorphose, in relation to the ideas of representation as well as of monsters, freaks of nature. It then analyses the strong link that inevitably develops between the evocation of metamorphosis and poetics, and finally brings out literary examples of transformations as variations on the body and its states.

Keywords : metamorphosis, monstrosity, poetics, body.

I

L NE SAURAIT être question ici de proposer une introduction à la littérature de métamorphoses dans les lettres et les arts de l'Espagne classique : une telle ambition ne pourrait être légitime et réaliste que dans le cadre d'un travail très vaste, fondé sur une étude précise de la réception de certains textes et de la survie - sous le mode de la reformulation, de la réappropriation, de la 
similitude et de la différence - de tout un ensemble de motifs que l'on pourrait nommer "métamorphiques ", déjà présents dans les textes de l'Antiquité. Notre propos se bornera ici à deux fonctions complémentaires : il s'agira tout d'abord de rendre compte et de tenter de justifier, au nom de l'association CRISOL 16/17, de notre choix thématique pour cette étude collective placée sous le signe de la métamorphose. S'ensuivront quelques considérations et exemples préliminaires qui constitueront un point de départ possible aux réflexions que le présent ouvrage rassemble.

\section{MÉTAMORPHOSE DES CORPS ET REPRÉSENTATION}

Le thème qui nous a réunis, de décembre 2009 à mars 2011, pour aboutir au présent ouvrage collectif, Métamorphose(s) : représentations et réécritures, nous a conduits à explorer sous l'angle de la représentation un premier domaine, celui du corps et de ses métamorphoses, et un deuxième, consacré aux transpositions artistiques et littéraires de ces métamorphoses. Ces deux concepts - corps, représentation - sont problématiques et nous incitent à une recherche sur les significations morales, spirituelles, philosophiques de certains phénomènes de bouleversement physique que l'on nomme, à juste titre ou non, métamorphoses. L'on peut affirmer que cette thématique est, d'une certaine manière, commune à bien des études sur le Siècle d'Or espagnol et sur les études littéraires de l'Europe moderne. En réalité, la question du corps, dans de nombreux écrits des $\mathrm{XVI}^{e}$ et XVII ${ }^{e}$ siècles, ne donne pas lieu à une approche univoque ou unique de ses contours, de ses aptitudes, de ses grandeurs et misères : doit-on, en suivant en cela la hiérarchie traditionnelle, entendre le corps comme la partie la plus faible et infirme de l'homme ? Est-il séparable d'une conception duale, binaire, qui investit l'âme de fonctions supérieures alors que le corps serait réduit au jeu des organes, à la répétition de fonctions vitales nécessaires, régulières et - disons-le - méprisables aux yeux des moralistes et théologiens ?

De nombreux travaux nous guident pour élaborer une première approche, notamment ceux publiés sous la direction d'Augustin Redondo dans deux volumes classiques intitulés respectivement Le corps dans la société espagnole des XVI et XVII siècles (1990) et Le corps comme métaphore dans lEspagne des $X V I^{e}$ et XVII siècles (1992). Le second volume entendait notamment - selon les termes d'Augustin Redondo - étudier scrupuleusement "le système de pensée analogique qui caractérise le Siècle d'Or ", dans lequel "de grandes correspondances existent entre l'univers (macrocosme), le corps humain (microcosme) et le corps de la république, "décalque" de ce dernier " ${ }^{1}$. Une telle configuration mentale donne lieu à une approche " organiciste " qui établit une série de correspondances entre les organes du corps et les différentes parties de

1. Augustin Redondo, "Introduction", Le corps comme métaphore dans l'Espagne des XVTe et $X V I I^{\mathrm{e}}$ siècles, études réunies et présentées par Augustin Redondo, Travaux du CRES [7], Paris, Publications de la Sorbonne Nouvelle, 1992, p. 5. 
la société et de l'État modernes, reproduction et prolongements profanes du corps christique que forme l'ensemble de la communauté des chrétiens. Au cours de ces quelques considérations préliminaires, les mutations corporelles qui vont retenir notre attention seront plus littérales que figurées, plus physiques et concrètes que métaphoriques. Cette approche corporelle nous est imposée par le sujet lui-même, par ce que signifie une métamorphose dans le contexte de la Renaissance qui est celui d'un renouvellement historique sans précédent de la conception du corps comme le précise Georges Vigarello dans son «Introduction" au premier volume de L'Histoire du corps :

C'est cette émergence du corps "moderne " qu'évoque d'abord ce livre : celui dont les dispositifs sont imaginés indépendamment de l'influence des planètes, de celle des forces occultes, amulettes ou objets précieux. Les mécanismes de ce corps se "désenchantent ", soumis à la vision nouvelle de la physique, expliqués par la loi des causes et des effets. Non que s'éloignent définitivement les croyances, celles de la médecine populaire, celles des sorciers de campagnes, celles des corps ployés par l'impensable. Non que disparaissent, loin s'en faut, les références sacrées. Longtemps, la vision banalisée du corps entremêle à son sujet toutes les influences, longtemps son enveloppe a semblé traversée par toutes les forces du monde. Mais un conflit de culture s'avive avec la Renaissance où le corps se singularise, spécifiant des fonctionnements expliqués par son propre « ressort » et par lui seul ${ }^{2}$.

Ce que (re)découvrent précisément non seulement les médecins et anatomistes mais également les penseurs, philosophes et écrivains du $\mathrm{XVI}^{e}$ siècle, c'est que le corps est un ensemble de correspondances, d'équilibres sans cesse en mouvement non seulement d'un point de vue humoral ${ }^{3}$ mais également anatomique. L'ouvrage qui constitue le point de départ de ce renouvellement est le De humani corporis fabrica d'Andrea Vesale (connu sous le nom latinisé d'Andreas Vesalius), publié à Bâle en 1543 et fondé sur une méthode expérimentale qui inclut la dissection d'êtres humains ${ }^{4}$. Son disciple espagnol, Juan Valverde de Hamusco, publia en 1556, sous le titre Historia de la composición del cuerpo humano, un traité d'anatomie dans lequel apparaît une partie des planches du traité vésalien ${ }^{5}$. Rafael Mandressi a par ailleurs consacré,

2. Georges Vigarello, «Introduction », dans Alain Corbin, Jean-Jacques Courtine et Georges Vigarello (sous la dir. de), Histoire du corps. 1. De la Renaissance aux Lumières, Paris, Seuil, 2005, p. 14.

3. En effet, comme le rappellent Roy Porter et Georges Vigarello, "L'équilibre humoral expliquait également le spectre des dispositions et des tempéraments : la complexion rougeaude de la personne généreusement dotée de sang, son entrain, son énergie, sa robustesse " (Ibid., p. 338). Avant d'ajouter : "La pensée humorale avait à sa disposition plusieurs explications du passage de la santé à la maladie. Tout allait bien lorsque les fluides vitaux coexistaient pacifiquement en bon équilibre de puissance : chacun était alors à sa mesure, adéquat aux fonctions corporelles permanentes, comme la digestion, la nutrition, la vitalité et l'évacuation des déchets. La maladie survenait lorsque l'une de ces humeurs s'accumulait (devenait "pléthorique"), ou s'asséchait " (Ibid., p. 338-339).

4. Andreas Vesalius, De humani corporis fabrica, Paris / Turin, Les Belles Lettres / Nino Aragno Editore, 2001. Cette très belle édition reproduit le fac-similé de la princeps de 1543.

5. Juan Valverde de Hamusco, Historia de la composición del cuerpo humano, Roma, Antonio 
voici quelques années, un ouvrage passionnant à la renaissance de l'anatomie, Le regard de lanatomiste ${ }^{6}$. Les œuvres d'Andrés de Laguna - traducteur de Dioscorides ${ }^{7}$ - et de Miguel Servet qui, le premier, a l'intuition du phénomène de la circulation sanguine dans son De motu sanguinis ${ }^{8}$, constituent deux autres témoignages de ce renouvellement majeur.

Parallèlement à cette réactivation de l'ancienne théorie des humeurs en physiologie, la psychologie va connaître certaines reformulations qui abondent également dans le sens d'une masse mouvante, changeante et ondoyante. Le modèle repose sur un principe premier, l'âme, qui connaît une grande diversité de bouleversements dont les traductions corporelles ou " somatisations " sont les affectus et sensations. Jean Starobinski l'étudie dans une synthèse importante intitulée "Brève histoire de la conscience du corps " (que je citerai d'après sa version française mais qui a été initialement publiée aux États-Unis dans Humanities Review) et l'expose de la manière suivante :

Pendant longtemps, la douleur et la volupté n'ont pas été attribuées à un système sensoriel particulier : elles sont dénommées " passions du corps » tandis que l'expression traditionnelle de "sens interne" (sensus internus) renvoie aux activités conscientes que l'esprit développe par et en lui-même (raison, mémoire et imagination), sur la base des informations fournies par les sens externes (vue, ouïe, goût, odorat et toucher). Selon la doctrine aristotélicienne, les données des sens externes ne parviennent au sens interne qu'après avoir été unifiées par le sens (sensorium commune, koinon aisthétérion). Le corps n'est nullement oublié : mais en tant que prévaut la médecine galénique, c'est principalement par la voie des humeurs, et non par une information nerveuse, qu'il est capable de modifier les activités de l'âme et d'être lui-même modifié en retour'.

Cette conscience, fortement influencée par le dogme antique et qui ne permet pas encore au corps de s'exprimer pleinement, de comprendre son propre caractère autonome, est profondément modifiée et renouvelée par le Traité des passions de lâme de René Descartes, qui paraît en $1644^{10}$. Entre ces deux termes et temps - le savoir antique et ses innombrables reformulations médiévales, le rationalisme cartésien qui exprime et façonne une nouvelle anthropologie - le danger est grand de ne penser l'époque que nous étudions que comme un entre-deux dans lequel il nous faudrait déceler des signes avantcoureurs de la révolution scientifique du milieu du XVII ${ }^{e}$ siècle. Il faut plutôt comprendre que cette incertitude paradigmatique, ce profond moment de

Salamanca y Antonio Lafreri, 1556. Nous avons pu travailler à partir du fac-similé suivant : Valladolid, Maxtor, 2005.

6. Rafael Mandressi, Le regard de l'anatomiste. Dissection et invention du corps en occident, Paris, Seuil, coll. « L'Univers Historique », 2003.

7. Pedacio Anazarbeo Dioscórides, Acerca de la medicina material y de los venenos mortíferos, traducción de Andrés Laguna, edición facsímil de la de 1555 (Amberes : Juan Latio), Madrid, Instituto de España, 1968-1969, 2 vols.

8. Miguel Servet, Obras completas. III. Escritos científicos, ed. de Ángel Alcalá, Zaragoza, Prensas de la Universidad de Zaragoza, Larumbe, coll. «Clásicos aragoneses », 2005.

9. Jean Starobinski, «Brève histoire de la conscience du corps », Revue française de psychanalyse, $1981, n^{\circ} 45 / 2$, p. 262.

10. Ibid., p. 262-263. Cf. René Descartes, Les passions de lâme, Paris, Jean Vrin, 1991. 
redéfinition, laisse précisément un champ considérable à différentes esquisses de reformulation et de réorganisation des savoirs corporels. Les exemples castillans ne manquent pas à cet égard, des plus illustres, comme l'Examen de ingenios de Huarte de San Juan (1575) ${ }^{11}$, aux plus méconnus comme la Nueva filosofía de la Naturaleza del Hombre de Oliva Sabuco de Nantes, traité publié en $1587^{12}$. Le premier livre, intitulé "Coloquia de el conocimiento de sí mismo " offre bel et bien une tentative de rénovation de l'analyse des différents affects de l'âme, qui préfigure ce que sera plus tard la psychologie d'abord cartésienne puis contemporaine. Curieusement ignoré en France, cet ouvrage vient de faire l'objet d'une traduction en anglais par des hispanistes de l'université de Chicago ${ }^{13}$.

Aussi - afin de clore cette première série de réflexions sur le corps - pourraiton donner quelques brefs exemples européens de l'usage des métamorphoses. Le plus présent à l'esprit du public français est sans nul doute celui de Montaigne qui a été amplement étudié, notamment par François Rigolot dans un livre très stimulant ${ }^{14}$, ainsi que par Jean Starobinski ${ }^{15}$. Si François Rigolot a pu étudier, dans son ouvrage, ce qu'il considère comme " une influence modélisante des Métamorphoses d'Ovide sur la poétique des Essais " ${ }^{16}$ afin de rendre compte de la "branloire pérenne » du monde, Jean Starobinski s’intéressa plus précisément à la critique de la médecine dans les Essais en montrant que " mon corps " individuel est incessible, inaliénable et doit avoir sa propre poétique, ce que contribuent à bâtir les Essais ${ }^{17}$. La notion de métamorphose est profondément attachée, liée à l'écriture et au corps comme s'il s'agissait d'une nécessité impérieuse. Un second exemple offre une conception encore plus radicale et fondamentale de la notion de "métamorphose » : celui de Giordano Bruno qui écrit, dans sa comédie le Chandelier: «Le temps ôte tout et donne tout; toutes choses se transforment, aucune ne s'anéantit ; l'un seul est immuable, l'un seul est éternel et peut demeurer éternellement semblable et même $~^{18}$. Yves Hersant précise au sujet de la conception de la nature et de l'homme selon Bruno :

Tel que le décrit le philosophe, notre monde physique est en transformation continuelle : il est en incessante créativité, une série infinie d'agrégations et de

11. Huarte de San Juan, Examen de ingenios [1575], ed. de Guillermo Serés, Madrid, Cátedra, coll. « Letras Hispánicas » [311], 1989.

12. Oliva Sabuco de Nantes, Nueva filosofia de la naturaleza humana, Madrid, Editorial Nacional, 1981.

13. Oliva Sabuco de Nantes Barrera, New Philosophy of Human Nature Neither Known to nor Attained by the Great Ancient Philosophers, Which Will Improve Human Life and Health, translated and edited by Mary Ellen Waithe, Maria Colomer Vintró and C. Angel Zorita, Chicago, University of Illinois Press, 2007.

14. François Rigolot, Les métamorphoses de Montaigne, Paris, PUF, coll. "Écritures ", 1988.

15. Jean Starobinski, Montaigne en mouvement, Paris, Gallimard, coll. "Folio Essai », 1993.

16. François Rigolot, op. cit., p. 218.

17. Jean Starobinski, "Le moment du corps ", Montaigne en mouvement, op. cit., p. 266-350.

18. Yves Hersant, " "Toutes choses se transforment" : Giordano Bruno et la métamorphose", dans Jackie Pigeaud (sous la dir. de), Métamorphose(s). XIII entretien de la Garenne Lémot, Rennes, PUR, 2010, p. 166. 
dissociations. Cette perpétuelle mutation de la nature, qu’il arrive à Bruno de présenter allégoriquement en Circé - assimilée à « la matière génératrice de toute chose » -, il ne faut pas la concevoir comme transformant une matière qui pour sa part serait stable : non moins que les formes, la matière est sujette à mutation ${ }^{19}$.

De ce même fait, «dans l'univers infini, pour être homme et l'être pleinement, il faut redevenir soi-même métamorphique " : c'est en se métamorphosant que l'homme s'accorde à la nature et à son flux dans la mesure où, comme l'explique Nuccio Ordine, "s'il n'est pas donné à l'homme d'agir dans la sphère de la nécessité, le domaine du modifiable constitue en revanche son terrain d'intervention ${ }^{20}$. Et c'est bien précisément cette volonté de peser sur son propre destin, de choisir sa propre forme, qui va pousser l'homme au choix de la métamorphose.

Cette diversité des regards portés sur le corps - que l'approche, comme on l'a esquissé, soit physiologique, anatomique, pathologique ou psychologique s'accompagne, d'une part, d'un intérêt très vif pour l'idée d'expérience et, d'autre part, d'une interrogation, rarement explicite, sur ce que peut un corps, sur ses aptitudes, ses capacités et ses limites. Cette série de mutations historiques de la conception du corps que nous venons d'évoquer ne constitue pas tant un " contexte » qui nous permettrait de comprendre le recours aux métamorphoses. Il convient également de souligner, dans ce processus de renouvellement de la vision du corps, l'importance décisive de l'intérêt pour les corps déformés et les différentes formes de monstruosités qui s'est manifesté dans toute l'Europe de la Renaissance, dans un discours qui oscille entre pseudo-raisonnement scientifique et développements invraisemblables. Qu'il s'agisse de Monstres et prodiges d'Ambroise Paré $^{21}$ - pourvu d'une riche iconographie - dont l'importance a été mise en valeur par les travaux de Jean Céard ${ }^{22}$ ou de l'un des nombreux recueils qui circulaient en Europe, la fascination pour l'anormalité et les différentes formes de démesure apparaît comme l'une des formes de la fascination pour la forme ondoyante des corps. Pierre Ancet a ainsi récemment pu mettre en lumière le caractère inséparable du monstre et du regard qui le considère, l'épouse, le jauge à l'aune d'une normalité fluctuante : de la "mise en scène " à la " déréalisation ", le monstre semble appeler la représentation et la prolifération discursive ${ }^{23}$. Un phénomène analogue se développe dans les pratiques artistiques des seizième et dix-septième siècles où la déformation adopte une série de formes inédites et gagne même la perspective qui connaît des altérations et de nouveaux jeux ${ }^{24}$.

19. Ibid., p. 170.

20. Ibid., p. 172.

21. Ambroise Paré, Monstres et prodiges, éd. de Jean Céard, Genève, Droz, coll. « Bibliothèque d'Humanisme et de Renaissance ", 1971.

22. Jean Céard, La nature et les prodiges, Genève, Droz, coll. « Titre Courant », 1996.

23. Pierre Ancet, Phénoménologie des corps monstrueux, Paris, PUF, 2007. On se reportera également au numéro très stimulant de la revue Imaginaire et inconscient consacré aux Représentations du monstrueux, 2004, $\mathrm{n}^{\circ} 13$.

24. Sur l'importance de la représentation monstrueuse dans l'histoire des arts occidentaux, 
Si l'on continue de se pencher sur le cas-limite (et hautement instructif) que constitue la représentation du monstrueux comme métamorphose du corps normal, il apparaitt que différentes logiques vont alors se manifester et tendre à offrir de nouveaux motifs à ce qui n'était auparavant qu'un ensemble de figures chimériques ou démoniaques. L’anormal semble en effet proliférer dans l'Espagne du Siècle d'Or, alimenter les débats et polémiques - naturalistes, philosophiques, voire linguistiques - et peupler les rues et les cités ${ }^{25}$. Cet univers monstrueux s'intègre dans un renouvellement esthétique sans précédent par lequel les formes prétendent incarner les métamorphoses du monde, le dédoublement des êtres et les équivocités des figures ${ }^{26}$. De cet ensemble d'une très grande richesse et diversité, nous ne souhaiterions retenir qu'un seul exemple, celui d'un monstre dont on a voulu à la fois établir l'existence d'un point de vue historique et déchiffrer les sens occultes que sa naissance impliquait. Il s'agit du monstre de Ravenne qui apparut en 1512 et dont la renommée se répandit dans toute l'Europe : en effet, dès 1512, Jean Multivallis le cita dans sa Chronique de l'histoire universelle, texte qui fut d'abord repris par Jacob Rueff dans son traité De conceptu et generationes hominis (1554) puis dans le fameux ouvrage de Lycosthène, Prodigiorum ac ostentorum chronicon (1557). Le monstre italien va atteindre le faite de la gloire avec les Histoires prodigieuses de Pierre Boaistuau, qui lui consacre le quarantième et dernier chapitre de son ouvrage $^{27}$. Aussi, lorsque Mateo Alemán recueille ce cas célèbre dans le premier chapitre du Guzmán de Alfarache, celui-ci a-t-il une longue histoire textuelle qui a fait de l'événement initial (si tant est qu'il ait réellement existé) une représentation d'une grande complexité et profondément binaire et équivoque à l'image de la vitalité démesurée du père du protagoniste-écrivain ${ }^{28}$.

Si l'on considère les quelques points que nous venons de développer, il en ressort que l'idée de monstruosité renvoie à une métamorphose du corps

l'ouvrage de Gilbert Lascault, Le monstre dans lart occidental, Paris, Klincksieck, 2004, demeure une référence incontournable par l'ampleur et la précision du panorama qu'il dresse. La question de la perspective a été traitée par Jurgis Baltrusaitis dans les deux premiers volumes de ses Perspectives dépravées: I. Aberrations, Paris, Flammarion, coll. "Champs-Flammarion », 1995 et II. Anamorphoses, Paris, Flammarion, coll. "Champs-Flammarion », 1996.

25. Elena del Río Parra, Una era de monstruos. Representaciones de lo deforme en el Siglo de Oro español, Madrid / Frankfurt an Main, Vervuert / Iberoamericana / Universidad de Navarra, 2003. Voir également l'étude de María José Vega, Los libros de prodigios en el Renacimiento, Bellaterra, Universidad de Barcelona, Publicaciones del Seminario de literatura medieval e hispanística, 2002.

26. Sur l'esthétique baroque, il convient de rappeler le caractère fondamental de l'étude de Jean Rousset, La littérature de l'âge baroque en France, Paris, José Corti, 1995 [1 [1 éd. 1953].

27. Pierre Boaistuau, Histoires prodigieuses, édition préfacée par Yves Florenne, Paris, Le Club français du livre, 1961, p. 308-310. Une traduction espagnole de ce texte, due à Andrea Pescioni, a circulé dès la fin du seizième siècle et a connu plusieurs rééditions.

28. Nous renvoyons le lecteur qui serait intéressé par ce "cas " à un texte où nous avions pu développer plus précisément la trame textuelle et analogique liée au monstre de Ravenne : cf. Philippe Rabaté, " Du portrait du père à l'évocation du monstre de Ravenne : la description chez Mateo Alemán (Guzmán de Alfarache, Première partie, I, 1) », dans Maria Aranda (sous la dir. de), Description et fiction de Jean de la Croix à Vargas Llosa, Rennes, PUR, 2008, p. 117-141. 
poussée à sa limite la plus extrême, celle d'une séparation radicale avec l'ordre normé des corps. Cette division ainsi instituée donne lieu à un véritable désir de représentation qui va adopter des formes extrêmement variées, aussi bien discursives qu'iconographiques. Comme on aura pu le comprendre, ce phénomène naît également de ce mouvement de renouvellement profond de la vision du corps qui apparaît avec la Renaissance. L'ouvrage de Michel Jeanneret offre précisément une étude globale sur la nature protéiforme de l'écriture et des arts de l'âge classique ${ }^{29}$. En ce sens, les métamorphoses ont tout leur sens dans cette lente découverte des pouvoirs du corps, dans cette écriture de l'expérience. Peut-on parler pour autant d'un modèle ou d'un paradigme d'écriture à l'âge moderne ?

\section{LES MÉTAMORPHOSES : CORPUS INITIAL ET MODÈLE POÉTIQUE}

Dans cette tentative pour écrire et décrire les états du corps, cette masse changeante et ondoyante de sensations, d'idées, les auteurs, sculpteurs, peintres de la Renaissance vont redécouvrir, également sur ce plan-là, les œuvres antiques. Dans un ouvrage classique, La survivance des dieux antiques, Jean Seznec a ainsi pu étudier " la métamorphose des Dieux » d'un point de vue textuel et, surtout, plastique $^{30}$. Il s'intéresse à toute une série de mutations formelles par delà le critère d'un respect pur et simple du style classique et, plus particulièrement, se penche sur les métamorphoses et avatars de ces dieux classiques, sous la forme de "ces dieux du Moyen-Âge, ces dieux-bâtards, ces dieux fantômes " ${ }^{31}$. Les réappropriations que nous offre Jean Seznec constituent tout un cycle déformant qui débouche sur le triomphe de formes classiques à la Renaissance. L'idéal formel ne s'est pas perdu et les altérations ont donc permis une survie des figures anciennes des Dieux. Ce processus ne serait-il pas similaire à celui que connaissent les textes littéraires ? Il est certain que l'opposition entre littérature médiévale et littérature renaissante et baroque a été trop souvent avancée de manière exagérée. Il semble que le corpus métamorphique se soit imposé comme l'une des sources d'écriture dès la fin du XV siècle. Sans vouloir trop détailler ce point, force est de constater que les quelques textes fondamentaux de la littérature anthropomorphique - à savoir, les Métamorphoses d'Ovide, L'Âne d'or d'Apulée et, dans une moindre mesure, L'Âne de Lucius de Patras ont connu des traductions - le plus souvent partielles, mais parfois intégrales avant même que n'ait débuté la Renaissance. L'Âne d'or d'Apulée connaît une première traduction de la main de Diego López de Cortegana dont la première

29. Michel Jeanneret, Perpetuum mobile. Métamorphoses des corps et des auvres de Vinci à Montaigne, Paris, Macula, 1997. Nous savons gré à Fernando Copello de nous avoir fait découvrir cet ouvrage et d'en avoir livré une analyse passionnante lors de l'une des séances de travail de CRISOL 16/17.

30. Jean Seznec, "La métamorphose des dieux ", La survivance des dieux antiques, Paris, Flammarion, coll. "Champs-Flammarion », 1993, p. 177-215.

31. Ibid., p. 177. 
édition est publiée à Séville dès $1513^{32}$ et qui va être rééditée à de nombreuses reprises tout au long du XVI ${ }^{e}$ siècle. Traduction élégante, fondée sur la version italienne de Béroalde de 1500, qui, du reste, est encore publiée de nos jours et dont Michèle Guillemont et Marie-Blanche Requejo Carrió ont souligné l'importance comme source des œuvres de Mateo Alemán et de Miguel de Cervantes ${ }^{33}$. Elle figure d'ailleurs dans les traductions castillanes reproduites et commentées par Juan Martos ${ }^{34}$. Dans le cas d'Ovide, il existait une tradition médiévale bien établie qui était celle de l'Ovide moralisé, probablement écrit au début du XIVe siècle et qui «moralise » l'œuvre ovidienne selon un processus de déformation très proche de ce qu'a pu étudier Jean Seznec dans La survivance des dieux antiques ${ }^{35}$. Cette survivance initiale va donner lieu à une première traduction castillane qui est pratiquement contemporaine de celle de L'Âne d'or: la traduction par Juan de Aguayo publiée également à Séville en 1518. En suivant les pas de l'ouvrage classique de Rudolph Schevill ${ }^{36}$, Aurora Egido reconstitue, dans un chapitre de son ouvrage El gran teatro de Calderón consacré aux figures d'Ulysse et de Circé, les différentes versions castillanes qui ont pu influencer les lettres espagnoles tout au long du seizième siècle ${ }^{37}$. À cette traduction - qui prend un assez grand nombre de libertés avec le texte ovidien mais qui connut trois rééditions sévillanes et une ultime réédition à Medina del Campo en 1540 -, succède celle, en prose, de Jorge Bustamante, publiée en 1546, d'une plus grande infidélité encore. Pour sa part, la version de Felipe Mey, qui voit le jour à Tarragone en 1586, ne reprend pas l'ensemble des contes ou fabulas. Enfin, pour clore ce survol très incomplet du XVI siècle, la traduction de Pedro Sánchez de Viana, intégrale et " en tercets et quatrains ", offre un véritable texte de référence mais qui ne sera pas réédité avant la fin du $\mathrm{XIX}^{\mathrm{e}}$ siècle $^{38}$.

Si ces quelques éléments historiques nous offrent de précieuses indications sur le phénomène éditorial que constituent ces nouvelles éditions, il convient à présent de se demander, de manière beaucoup plus formelle, si la forme poétique

32. Apuleyo, El asno de oro, traducción del Licenciado Diego López de Cortegana, Sevilla, Jacopo Cromberger, 1513.

33. Michèle Guillemont, Marie-Blanche Requejo Carrió, « De asnos y rebuznos. Ambigüedad y modernidad de un diálogo ", Criticón, 101, 2007, p. 57-87. Sur Cortegana, voir plus précisément les pages 60-61.

34. Apuleyo, Las metamorphosis o el Asno de Oro. Apuleyo de Madauros, ed. de Juan Martos, Madrid, CSIC, 2003, 2 vols.

35. Catherine Croizy-Naquet, «L'Ovide moralisé ou Ovide revisité : de métamorphose en anamorphose ", Cahiers de recherches médiévales et humanistes, 9, 2002, mis en ligne le 05 janvier 2007. URL : http://crm.revues.org//index49.html.

36. Rudolph Schevill, Ovid and the Renaissance in Spain, Berkeley, University of California Press, 1913.

37. Aurora Egido, "La fábrica de un auto : Los encantos de la culpa", dans El gran teatro de Calderón : personajes, temas y escenografía, Kassel, Reichenberger, 1995, p. 87-98.

38. Las transformaciones de Ovidio, traducción del Licenciado Diego Sánchez de Viana, Valladolid, Diego Fernández de Córdoba, 1589. La version numérisée de ce texte est disponible sur le site de l'université de Toulouse-II. 
de la métamorphose a pu constituer un modèle d'écriture et à quels traits ou à quelles caractéristiques nous le pourrions réduire. Quelle peut être la légitimité d'une vaste synthèse historique qui prétendrait étudier la diachronie du genre sur près d'une vingtaine de siècles ${ }^{39}$ ? Dès les premiers vers des Métamorphoses, Ovide nous annonce ce qu'il entend chanter, la métamorphose ou changement de forme et d'apparence des corps :

J'ai formé le dessein de conter les métamorphoses des êtres en des formes nouvelles. Ô dieux (car ces transformations furent, elles aussi, votre œuvre), favorisez mon entreprise et guidez le déroulement ininterrompu de mon poème depuis l'origine même du monde jusqu'à ce temps qui est le mien ${ }^{40}$.

Ce changement de forme est absolument consubstantiel à la fable et en détermine les différents aspects poétiques, même s'il demeure assez rare de voir la description de la métamorphose comme processus en train de se faire, comme métamorphose en acte. Dans sa thèse de doctorat d'état ${ }^{41}$, Gilles Tronchet tente d'étudier à la lumière de la narratologie de Gérard Genette les récits ovidiens et définit de la sorte une "métamorphose »:

Je retiendrai donc d'emblée comme métamorphose toute transformation surnaturelle affectant l'apparence d'un être, que cela résulte d'une intervention extérieure ou bien d'une mutation interne. Une telle approche permet d'envisager un éventail assez large de légendes, qui abritent les aspects les plus familiers du motif, lorsque la figure humaine devient source ou rocher, plante ou animal, selon une diversité facile à répartir en quelques grandes catégories ${ }^{42}$.

Cette mutation implique donc une antériorité et une postériorité, un corps ancien et un corps nouveau dont l'apparition peut être imputable à l'action des Dieux ou à un devenir corporel propre. Ce changement peut être unique et définitif ou bien peut révéler - chez un personnage tel que Protée - «le don de revêtir une série de formes successives " ${ }^{43}$. Pour sa part, Michel Le Guern, dans une tentative de définition de la métamorphose poétique, a rappelé à quel point cette constance thématique était inséparable de traits de composition permanents : «On distingue dans la métamorphose quatre parties principales : l'exposition, la narration, l'incident et la conclusion $»^{44}$. Toutefois, une telle définition ne saurait suffire et nous conduit à ne considérer que le corpus ovidien

39. L’on citera, à cet égard, la tentative récente de Gregorio Cabello Porras et de Francisco Javier Campos Daroca (coord.), Poética de la metamorfosis : tradición clásica, Siglo de Oro y modernidad, Málaga, Universidad de Málaga, coll. «Thema », 2002.

40. Ovide, Métamorphoses, Introduction, traduction et notes de Joseph Chamonard, Flammarion, coll. "GF-Flammarion », 1966, livre premier, p. 41.

41. Gilles Tronchet, La métamorphose à louvre. Recherches sur la poétique dOvide dans les "Métamorphoses ", Louvain, Peeters, 1998.

42. Ibid., p. 198.

43. Ibid., p. 199.

44. Michel Le Guern, «La métamorphose poétique : essai de définition », dans Guy Demerson (sous la dir. de), Poétiques de la métamorphose, Saint-Étienne, Publications de l'université de Saint-Étienne, 1981, p. 27. 
ainsi que celui de ses plus proches imitateurs et continuateurs. Les analyses de Gilles Tronchet et de Michel Le Guern concordent de manière très significative sur un point, qui est que les métamorphoses délimitent et célèbrent le royaume immense de la similitudo, de l'analogie et de la correspondance. C'est peut-être précisément à cause de cette recherche d'un réseau de similarité que l'on peut parler d'un modèle d'écriture qui s'inscrit dans le champ des possibles comme le remarque Michel Le Guern :

Le poète doit aussi révéler ce qui est caché. Il dira les transformations qui échappent aux sens, en les projetant dans l'ordre du sensible grâce à la similitude. Les habitudes $\mathrm{du} \mathrm{XVI}{ }^{\mathrm{e}}$ siècle sont particulièrement favorables à un tel outil logico-sémantique de la comparaison-image. Là où elle n'est plus pour nous qu'image, elle était bien souvent argument ${ }^{45}$.

Avant de conclure :

C'est parce qu'elle s'inscrit dans ce système d'analogies que la correspondance entre une transformation visible et une transformation invisible permet de dire celle-ci en lui prêtant une épaisseur concrète qui la rend en quelque sorte visible elle aussi ${ }^{46}$.

Les métamorphoses relèvent ainsi de la création de réseaux d'analogie mais elles doivent être également lues et interprétées de manière sérielle, chaque métamorphose intégrée dans une ouvre entrant en résonance avec l'ensemble des métamorphoses qui y figurent. Aussi les métamorphoses mythologiques vont-elles tout particulièrement retenir l'intérêt des écrivains mais aussi des mythographes dans la mesure où il s'agit en effet d'un réseau de figures déjà constitué, et apte à recevoir de nouvelles reformulations. De la sorte, nous souhaiterions évoquer, avant de clore ce propos préliminaire, les liens qui unissent la logique vitale et la chronologie de l'écriture - en d'autres termes le déploiement de la métamorphose - avant de proposer quelques brèves suggestions sur la signification morale des différents états métamorphiques à travers un culte de la forme, entre dégénérescence et progrès.

\section{LOGIQUe VITALE ET CHRONOLOGIE DE L'ÉCRITURE : LE DÉPLOIEMENT DE LA MÉTAMORPHOSE}

Comme le constate Jackie Pigeaud, «l'une des difficultés les plus grandes, dans l'imagination de la métamorphose, du point de vue de la représentation plastique comme du point de vue du discours, de l'ekphrasis, est de rendre le temps de la métamorphose, et de la montrer en acte $»^{47}$. Gregorio Cabello Porras et Francisco Javier Campos Daroca remarquent pour leur part : "Metamorfosis designa una perspectiva por la cual el cambio es remitido tozudamente a la

\footnotetext{
45. Ibid., p. 28.

46. Id.

47. Jackie Pigeaud, "Ouverture », Métamorphose(s), op. cit., p. 13.
} 
continuidad, como si en cada paso de la transformación nos forzáramos a ver, avanzando a traspiés, el paso anterior y, al término, nos volviéramos al punto de partida con el dolor de la pérdida $"^{48}$. Il est en effet malaisé de montrer une telle transformation dans son inachèvement, dans son déploiement et nous voudrions consacrer quelques réflexions à ce processus en évoquant plusieurs exemples classiques. Le lecteur du Siècle d'Or pensera très probablement à l'un des sonnets les plus fascinants de Garcilaso de le Vega qui reprend le mythe d'Apollon et de Daphné. Il s'agit du sonnet XIII qui est un magnifique exemple de métamorphose corporelle en acte :

A Dafne ya los brazos le crecian $y$ en luengos ramos vueltos se mostraban; en verdes hojas vi que se tornaban los Cabellos qu'el oro escurecian;

de áspera corteza se cubrian
los tiernos miembros que aun bullendo 'staban
los blancos pies en tierra se hincaban
y en torcidas raices se volvian.

Aquel que fue la causa de tal daño, a fuerza de llorar, crecer hacía este árbol, que con lágrimas regaba.

\footnotetext{
¡Oh miserable estado, oh mal tamaño, que con llorarla crezca cada día la causa y la razón por que lloraba. ${ }^{49}$
}

Nous nous bornerons à deux remarques très brèves et renverrons le lecteur, pour une analyse infiniment plus précise de l'œuvre garcilasienne, à la contribution de Juan Diego Vila incluse dans le présent volume. En premier lieu, il convient de remarquer que l'ensemble de l'écriture est fondé sur une chronologie dont le point central est la métamorphose se faisant, membre par membre, avec - si l'on reprend les catégories aristotéliciennes - le passage du paradigme de la vie humaine à la vie végétative. À cette transformation, s'ajoute l'impuissance du Dieu innommé - Apollon - qui s'apparente à une figure du poète impuissant, ce que le texte énonce à l'aide d'un paradoxe qui s'exprime dans les tercets. Les pleurs d'Apollon sont une invention garcilasienne là où prédominait, dans le texte ovidien, le récit de la transformation et la représentation de l'être hybride :

À peine sa prière achevée, voici qu'une pesante torpeur envahit ses membres; sa tendre poitrine est enveloppée d'une mince écorce, ses cheveux s'allongent en feuillage, ses bras en rameaux, son pied, tout à l'heure si rapide, est retenu au sol par d'inertes racines ; son visage, à la cime, disparaît dans la frondaison. Seul subsiste en elle l'éclat

48. Gregorio Cabello Porras, Francisco Javier Campos Daroca, "Introducción ", Poética de la metamorfosis: tradición clásica, Siglo de Oro y modernidad, op. cit., p. 7.

49. Garcilaso de la Vega, "Soneto XIII ", Poesías castellanas completas, ed. de Elias L. Rivers, Madrid, Castalia, Clásicos Castalia, 1996, p. 5. 
de son charme. Telle, Phoebus l'aime encore, et sa main posée sur le tronc sent le cœur qui continue à battre sous la neuve écorce. Entourant de ses bras, comme des membres, les branches, il couvre de baisers le bois; mais le bois se dérobe à ses baisers ${ }^{50}$.

Comme on le sait, Garcilaso offre une autre version de ce même mythe du désir inassouvi et de la transformation de l'objet aimé - empreint de résonances personnelles et d'une hantise face à l'absence - dans la Égloga tercera, sous la forme, cette fois-ci, d'une ekphrasis ${ }^{1}$. Le même motif du planctus divin réapparait au sein d'une écriture qui choisit de faire figurer l'ensemble de la chronologie esquissée par Michel le Guern - exposition, narration, incident et conclusion - avec un chevauchement de certaines de ces fonctions. Le déploiement de la métamorphose se dessine ainsi clairement et le mythe repris se voit assigner de nouvelles significations. Juan Pérez de Moya en livrait une interprétation polysémique dans sa Philosophía secreta de la gentilidad publiée en 1585 : après une brève narration - fondée sur une compilation des différentes autorités - il se livre à une double interprétation qui repose sur le "sentido natural " (" humidad" opposée au "sol» de Apolo ${ }^{52}$ ) et "moral " : Por esta fábula quisieron los antiguos loar la castidad, fingiendo que los que la guardaban se

50. Ovide, Les métamorphoses, I, v. 472-578, trad. citée, p. 57.

51. Garcilaso de la Vega, "Égloga tercera ", Poesía castellana completa, op. cit., v. 145-168, p. 215-216:

\author{
Dinámene no menos artificio \\ mostraba en la labor que habia tejido, \\ pintando a Apolo en el robusto oficio \\ de la silvestre cabeza embebecido. \\ Mudar presto le hace el ejercicio \\ la vengativa mano de Cupido, \\ que hizo a Apolo consumirse en lloro \\ después que le enclavó con punta d'oro. \\ Dafne, con el cabello suelto al viento, \\ sin perdonar al blanco pie corría \\ por áspero camino tan sin tiento \\ que Apolo en la pintura parecia \\ que, porqu'ella templase el movimiento, \\ con menos ligereza la seguia; \\ él va siguiendo, y ella huye como \\ quien siente al pecho el odioso plomo. \\ Mas a la fin los brazos le crecian \\ $y$ en sendos ramos vueltos se mostraban; \\ y los cabellos, que vencer solian \\ al oro fino, en hojas se tornaban; \\ en torcidas raices s'estendian \\ los blancos pies y en tierra se hincaban; \\ llora el amante y busca el ser primero, \\ besando y abrazando aquel madero.
}

52. Juan Pérez de Moya, Philosophía secreta de la gentilidad, ed. de Carlos Clavería, Madrid, Cátedra, coll. "Letras Hispánicas ", 1995, p. 268. Sur la constitution du discours amoureux profane et sacré et ses soubassements médicaux, on se reportera également à l'ouvrage classique de Guillermo Serés, La transformación de los amantes, Barcelona, Crítica, 1996. 
convertian en árboles siempre verdes, como Daphne en laurel y Lotos en otro árbol asi llamado, dando a entender por estos árboles la virtud de la castidad " ${ }^{53}$.

Cette transformation, dépeinte minutieusement, peut également être l'un des recours a lo divino pour signifier l'amour de Dieu dans une perspective mystique. Pour ne retenir qu'un seul exemple et de manière assez fugace, nous citerons le célèbre poème de saint Jean de la Croix, " noche escura ", qui offre cette même chronologie de la transformation en un sens mystique :

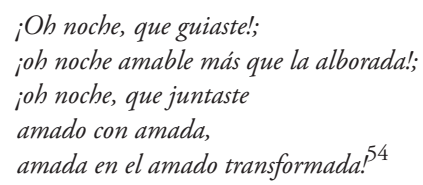

53. Ibid., p. 269.

54. Juan de la Cruz, Obras completas, ed. de Lucinio Ruano de la Iglesia, Madrid, BAC, 2005, p. 107. Voici l'intégralité du texte (Ibid., p. 106-107):

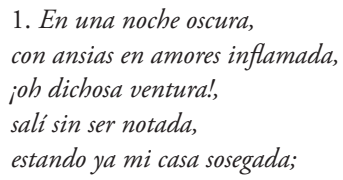

1. En una noche oscura,

con ansias en amores inflamada,

joh dichosa ventura!,

sali sin ser notada,

estando ya mi casa sosegada;

2. a oscuras y segura,

por la secreta escala, disfrazada,

¡oh dichosa ventura!

a oscuras y encelada,

estando ya mi casa sosegada;

3. en la noche dichosa,

en secreto, que nadie me veía

ni yo miraba cosa,

sin otra luz y guía

sino la que en el corazón ardía.

4. Aquesta me guiaba

más cierto que la luz del mediodía,

adonde me esperaba

quien yo bien me sabia,

en parte donde nadie parecía

[...]

6. En mi pecho florido,

que entero para él sólo se guardaba,

alli quedó dormido,

y yo le regalaba,

y el ventalle de cedros aire daba.

7. El aire del almena,

cuando yo sus cabellos esparcía,

con su mano serena

en mi cuello hería,

y todos mis sentidos suspendía.

8. Quedéme y olvidéme,

el rostro recliné sobre el Amado,

cesó todo y dejéme,

dejando mi cuidado

entre las azucenas olvidado. 
Ce poème, qui s'inscrit dans la tradition du Cantique des cantiques, traduit et commenté à la même époque par Fray Luis de León ${ }^{55}$, décrit la transformation de l'âme dans l'union avec Dieu et participe d'un vaste mouvement de réappropriation des métamorphoses par le discours religieux.

Le recours à l'écriture métamorphique, au dévoilement d'un sens caché ou latent par le biais d'une transformation corporelle va devenir l'un des lieux communs les plus récurrents de la littérature que l'on appelle baroque. L'espace nous manque dans le cadre de ce propos préliminaire mais il conviendrait d'étudier scrupuleusement sa présence dans le théâtre caldéronien, dans les textes satiriques quévédiens - notamment les Sueños ou, de manière encore plus affirmée, dans La hora de todos - et nous pourrions mesurer l'importance d'une allégorisation du discours de la transformation corporelle, déjà présente chez Juan Pérez de Moya. Nous nous bornerons à mentionner l'un des exemples les plus saisissants de cette écriture métamorphique qu'est le grand récit allégorique de Baltasar Gracián, El Criticón (1651-1657). Ainsi, dans « Los encantos de Falsirena ", le portrait de Falsirena en Circé des temps modernes entraîne toute une série de métamorphoses enchaînées qui montre la puissance visuelle du mal, de l'altération :

-No os canséis ni recibáis enfado. Es verdad que ha vivido abi algunos días una Circe en el zurcir y una sirena en el encantar, causa de tantas tempestades, tormentos y tormentas, porque a más de ser ruin, aseguran que es una famosa hechicera, una célebre encantadora, pues convierte los hombres en bestias; y no los transforma en asnos de oro, no, sino de su necedad y pobreza. Por esa corte andan a millares convertidos (después de divertidos) en todo género de brutos. Lo que yo sé decir es que, en pocos días que aqui ha estado, he visto entrar muchos hombres y no he visto salir uno tan sólo que lo fuese. Y por lo que esta sirena tiene de pescado, les pesca a todos el dinero, las joyas, los vestidos, la libertad y la honra; y para no ser descubierta, se muda cada día, no en la condición ni en las costumbres, sino de puestos: del un cabo en la villa salta al otro, con lo cual es imposible hallarla, de tan perdida. [...] Muda tantos nombres, como puestos. En una parte es Cecilia, por lo cila, en otra Serena por lo sirena, Inés porque ya no es, Teresa por lo traviesa, Tomasa por lo que toma y Quiteria por lo que quita. Con estas artes los pierde a todos, y ella gana y ella reina ${ }^{56}$.

Falsirena est donc, comme l'indique le programme onomastique contenu dans son nom, une Circé négative des temps modernes et elle occupe une place déterminante dans les villes que traversent les deux pèlerins à la fin de cette Première partie. Au sein d'une réécriture des " arts de naviguer " courtisans destinés aux jeunes nobles, et qui avaient proliféré au long du Cinquecento et du $\mathrm{XVI}^{\mathrm{e}}$ siècle castillan, la figure de Falsirena permet de concentrer un ensemble de figures et d'emblèmes répulsifs pour l'homme de raison : la dégradation de son corps, l'altération de ses capacités et de ses humanités, l'oubli de soi et de l'exigence d'être une persona, tentations auxquelles est soumis Andrenio de

55. Fray Luis de León, El Cantar de los cantares de Salomón, ed. de José María Becerra Hiraldo, Madrid, Cátedra, coll. « Letras Hispánicas », 2003.

56. Baltasar Gracián, «Los encantos de Falsirena », El Criticón, ed. de Santos Alonso, Madrid, Cátedra, coll. «Letras Hispánicas », 1980, I, 12, p. 254-255. 
manière incessante avant qu'il n'ait atteint un degré d'éducation et de culture suffisant pour savoir déjouer les pièges mondains. Face à ces métamorphoses négatives, celles que réalise sa rivale Artemia sont positives, comme si la pensée de Gracián ne pouvait qu'être " januale »-selon le terme employé par Aurora Egido dans un ouvrage de référence sur l'œuvre de Baltasar Gracián ${ }^{57}$. Aussi peut-on lire de manière contrapuntique ce portrait d'Artemia :

Érase una gran reina, muy celebrada por sus prodigiosos hechos, confinante con este primer rey, y por el consiguiente tan contraria suya que de ordinario traían guerra declarada y muy sangrienta. Llamábase aquélla, que no niega su nombre ni sus hechos, la sabia y discreta Artemia, muy nombrada en todos siglos por sus muchas y raras maravillas; si bien se hablaba de ella con grande variedad, porque aunque los entendidos sentían ( $y$, entre ellos, el primero el tan valeroso como discreto duque del Infantado) de sus acciones como quien ellos son y ella merece, pero lo común era decir ser una valiente maga, una grande hechicera, aunque más admirable que espantosa. Muy diferente de la otra Circe, pues no convertía los hombres en bestias, sino al contrario, las fieras en hombres. No encantaba personas, antes las desencantaba.

Artemia emploie son talent d'une manière telle que la portée éthique et la manifestation esthétique se mêlent en une seule et unique forme artistique d'être-au-monde :

De los brutos hacia hombres de razón; y habia quien aseguraba haber visto entrar en su casa un estólido jumento, y dentro de cuatro días salir hecho persona. De un topo hacer un lince era fácil para ella; convertía los cuervos en cándidas palomas, que era ya más dificultoso, asi como hacer parecer leones las mismas liebres, y águilas los tagarotes; de un búho hacia un jilguero. [...]

Daba vida a las estatuas y alma a las pinturas: hacia de todo género de figuras y figurillas, personas de substancia ${ }^{58}$.

L’on pourrait ainsi distinguer trois niveaux dans cette longue réécriture du mythe de Circé, hérité à la fois d'Homère et d'Ovide : après une présentation initiale d'Artemia comme une Circé bienfaisante, Gracián célèbre son pouvoir de reine des métamorphoses en évoquant à quel point celui-ci s'étend à l'ensemble des espèces vivantes et, enfin, son action " artistique " qui vise à conférer aux différents hommes la forme humaine la plus achevée. Nous nous trouvons ici devant un magnifique exemple d'utilisation allégorique des métamorphoses et, bien plus encore, face à l'un des moments de la constitution d'une philosophie de la métamorphose et de l'inversion. Il ne saurait être ici question de souligner à quel point la Circé de Gracián se différencie de celle évoquée par Pérez de Moya - vision qui se limite à définir ce personnage comme " aquella pasión natural que llaman amor deshonesto, que las más veces transforma a los más sabios y de mayor juicio en animales fierísimos y llenos de furor, y algunas veces los vuelve más insensibles que piedras, acerca de la honra $y$ reputación que conservaban con tanta diligencia antes que se dejasen cegar desta

57. Aurora Egido, Las caras de la prudencia y Baltasar Gracián, Madrid, Castalia, coll. « Nueva Biblioteca de Erudición y Crítica », 2000.

58. Baltasar Gracián, «Las maravillas de Artemia », El Criticón, op. cit., I, 8, p. 171-172. 
fierísima pasión " 59 . La représentation d'Artemia implique également une transformation du protagoniste qui doit suivre les pas d'Ulysse, que ce soit sur un terrain littéral ou figuré (autrement dit, allégorico-moral), et suivre par conséquent une trajectoire de métamorphose que Pietro Citati, dans un livre stimulant - La pensée chatoyante. Ulysse et lOdyssée - a évoquée de la manière suivante :

Ulysse est le seigneur des métamorphoses : il se masque et se transforme comme les dieux, comme Protée surtout, son équivalent dans les eaux originelles, lequel devient, en quelques instants, lion et serpent, panthère, sanglier, arbre et eau. Tantôt Ulysse se déguise en serviteur pour découvrir les secrets de Troie, tantôt il se fait mendiant, ou œuvre d'art entre les mains d'Athéna; ou bien encore héros épique, ou interprète des rêves. $[\ldots]$

Ainsi, à force de transformations et de secrets, à force de se tourner de tous côtés pour révéler tantôt un visage, tantôt le visage opposé, la nature d'Ulysse devient la plus vaste que nous ayons connue. On ne peut la circonscrire, parce qu'elle est toujours ailleurs. Alors qu'Achille se concentre sur lui-même, Ulysse se dilate à l'infini ${ }^{60}$.

Dans les trois types de discours littéraires que nous venons d'évoquer très brièvement - la lyrique amoureuse, la poésie de l'union mystique et la fable allégorico-morale -, l'on retrouve donc à des titres divers, une poétique de la métamorphose fondée sur la réappropriation de sa chronologie et de son déploiement, sans que nous ayons pu encore avancer d'hypothèses précises en ce qui concerne les différents états métamorphiques et le sens moral que le lecteur est invité à leur donner. C'est à cette interrogation que nous allons consacrer nos dernières remarques.

\section{De QUelQues ÉTATS MÉTAMORPHIQUeS : LA FASCINATION POUR LA FORME, ENTRE DÉGÉNÉRESCENCE ET REMÉDIATION MORALE}

Depuis les années 1530 , le renouvellement de la prose castillane, sous l'influence de la littérature italienne, privilégie différents états métamorphiques de l'homme qui relèvent de la dégradation et de l'altération corporelle. Qu'il s’agisse du Crotalón de Cristóbal de Villalón ou des premiers romans picaresques - le Lazarillo et bien davantage encore sa Seconde partie de 1555 qui met en scène la métamorphose de Lazare en thon -, les changements d'enveloppe corporelle s’imposent comme un recours narratif fréquent qui semble permettre de révéler un sens caché de la nature humaine. Le Guzmán de Alfarache constitue l'un des textes les plus marquants de ce courant négatif de représentation du corps.

59. Juan Pérez de Moya, Philosophía secreta de la gentilidad, op. cit., p. 547.

60. Pietro Citati, La mente colorata. Ulisse e l'Odissea, trad. française La pensée chatoyante. Ulysse et l'Odyssée par Brigitte Pérol, Paris, L’Arpenteur, 2004, p. 109-110. Sur la figure de Circé, on se reportera à l'article de Nadine Ly, «CIRCÉ(S) : De quelques figures féminines de Lope de Vega ", La femme dans la littérature et l'iconographie du Siècle d'Or : Vénus, Eve, Marie... ?, textes réunis par Nathalie Dartai-Maranzana et Emmanuel Marigno, Crisoladas, 2, 2007, p. 14-41. 
Il s'agit, comme on le sait, d'un texte profondément marqué par l'imaginaire humaniste : en ce sens, le parcours du protagoniste est présenté et décrit comme une dégradation formelle, qui ne verse cependant pas dans la représentation allégorique comme le font, peu après, les œuvres de Quevedo ou de Gracián. L'épaisseur même du corps - organes, sens, fluides - est entrevue par Alemán d'un point de vue médical et symptomatique ${ }^{61}$. L'importance de ces phénomènes d'altération nous place face à une série d'anecdotes ou d'aventures qui pourraient paraître purement et strictement passagères, sans conséquence durable sur le corps : en réalité, la métamorphose corporelle apparait, dans son sens le plus médical, comme l'un des symptômes du mal. On se souvient, à cet égard, des deux premières bourles que subit Guzmán lors de ses rencontres successives avec deux aubergistes peu scrupuleux : en guise de repas, la vieille aubergiste - première hôte du protagoniste - lui offre "un emplasto de huevos " ${ }^{62}$ qui est à l'origine de l'écœurement du personnage ; après avoir repris la route, il est en proie à un malaise physique évoqué non sans humour : "Así prosegui mi camino, y no con poco cuidado de saber qué pudiera ser aquel tañerme castañetas los huevos en la boca " ${ }^{63}$. La nourriture dévorée reprend donc une vie, ressuscite et acquiert une existence fantomatique et plaintive : "Y aun el día de hoy me parece que siento los pobrecitos pollos piándome acá dentro " ${ }^{64}$. L'expérience de la première auberge nous confronte en outre à un rapport bouleversé des frontières qui séparent communément la vie et la mort, le couvé et le non couvé, l'œuf - état embryonnaire - et l'animal déjà individué ; un tel dérèglement des repères vitaux essentiels s'accompagne, comme l'a montré Pedro Córdoba, de la négation d'un des interdits les plus fondamentaux de notre civilisation, la consommation d'êtres hybrides ${ }^{65}$. Outre la violation de ce tabou, cette anecdote constitue la première formulation explicite d'une altération qui est un préalable indispensable à la découverte d'une vérité :

Fui dando y tomando en esta imaginación, que, cuanto más la seguía, más géneros de desventuras me representaba y el estómago se me alteraba [...].

61. Pour une étude précise et stimulante des états du corps guzmanien et de l'importance du modèle médical, on se réferera à l'étude fondamentale de Francisco Ramírez Santacruz, El diagnóstico de la humanidad por Mateo Alemán: el discurso médico del "Guzmán de Alfarache», con un prólogo de Francisco Márquez Villanueva, Potomac, Scripta Humanistica [156], 2005.

62. Mateo Alemán, Guzmán de Alfarache, ed. de José María Micó, Madrid, Cátedra, coll. "Letras hispánicas ", 1987, I, p. 168. Nous citerons dorénavant toujours à partir de cette édition.

63. Ibid., I, p. 172-173.

64. Ibid., I, p. 173.

65. Pedro Córdoba, «À table avec Guzmán. Ethnologie du roman picaresque », Hommage à Robert Jammes, Toulouse, Presses Universitaires du Mirail, Anejos de Criticón [1], 1994, vol. I, p. 249-262. Il précise en effet à propos des œufs couvés et du mulet : « [...] si ces plats sont donc pour nous objet d'un tabou alimentaire, c'est bien évidemment, comme toujours lorsqu'il s'agit d'un tabou, pour des raisons culturelles et uniquement pour cela. Nous ne mangeons pas d'œufs couvés ni de mulet pour le même motif que Juifs et Musulmans refusent de manger du cochon. Exactement pour le même motif : parce qu'il s'agit d'hybrides » (la citation, p. 253-254). 


\begin{abstract}
Entre unas y otras imaginaciones encontré con la verdady, teniendo andada otra legua, con sólo aquel pensamiento, fue imposible resistirme ${ }^{66}$.
\end{abstract}

Cette prise de conscience à partir d'une douleur physique débouche sur le dénouement de la crise, qui se traduit par ce que l'on pourrait appeler une féminisation du personnage, métamorphose éphémère aussi soudaine qu'inattendue : "Porque, como a mujer preñada, me iban y venían eruptaciones del estómago a la boca, hasta que de todo punto no me quedó cosa en el cuerpo $"^{67}$. Comme le remarquait Francisco Márquez Villanueva dans un travail pionnier sur le savoir médical alémanien :

Dicha capacitación profesional de Mateo Alemán no debe de sorprender a nadie ni ofrece en si dificultad alguna. Hijo de un conocido galeno de la cárcel real de Sevilla, habia realizado estudios de medicina primero en su ciudad natal y después en Alcalá, donde al final de éstos le negaron el grado por su pública mácula de linaje judio. Añadió así la medicina a la cadena de frustraciones que marcó toda su vida, en la cual trató igualmente de abrirse camino como burócrata, comerciante, juez y, por último, poeta. Su obra entera es la de un médico que no puede menos de desarrollar una visión nosológica de la humanidad, cuyos vicios y defectos continuamente denuncia en términos de accidentes patológicos (llagas, cánceres, vómitos, etc.), conforme a una constante estilística en que la crítica aun no ha reparado ${ }^{68}$.

Ces différents traits qui apparaissent lors de la première déconvenue de Guzmán resurgissent avec plus d'intensité lors de son séjour chez le second aubergiste. Guzmán se présente à nouveau dans un véritable état d'exténuation :

Yo llegué tal de molido, que, dando con mi cuerpo en el suelo, no me pude rodear por muy gran rato. Llegué los muslos resfriados, las plantas de los pies hinchadas de llevarlos colgando y sin estribos, las asentaderas batanadas, las ingles dolorosas, que parecía meterme un puñal por ellas, todo el cuerpo descoyuntado y, sobre todo, hambriento ${ }^{69}$.

Il n'est pas nécessaire de citer la seconde bourle qui, avec la tromperie du mulet, achèvera la transformation de Guzmán et sa métamorphose en âne, "asnificación negativa " selon Marie-Blanche Requejo Carrió et Michèle Guillemont $^{70}$. On pressent bien que de telles métamorphoses peuvent être investies d'un sens moral et allégorique qui va se confirmer et prendre une vaste ampleur dans les lettres espagnoles, notamment à travers les Sueños et $\mathrm{La}$ hora de todos de Quevedo (que l'on se souvienne des figuras si présentes dans ses écrits), ainsi que l'œuvre de Gracián que nous avons déjà évoquée. Toutefois, la vision alémanienne ne peut-elle être que négative, ne peut-elle présenter qu'une vision dégradée du corps ? L’on se souvient qu'Alemán a même recours à une

66. Mateo Alemán, Guzmán de Alfarache, éd. citée, I, p. 173.

67. Id.

68. Francisco Márquez Villanueva, "El canto del cisne de Mateo Alemán: los Sucesos de fray García Guerra (1613)", Inquisición y conversos. III Curso de cultura hispano-judía y sefardi, Madrid, Asociación de Amigos del Museo Sefardí / Caja de Castilla-la-Mancha, 1994, p. 246.

69. M. Alemán, Guzmán de Alfarache, éd. citée, I, p. 189.

70. Marie-Blanche Requejo Carrió, Michèle Guillemont, « De asnos y rebuznos », art. cité, p. 61-65. 
figuration de la monstruosité à plusieurs reprises dans son œuvre et tend à faire de celle-ci une catégorie mobilisable pour asseoir le discours didactico-moral de l'œuvre ${ }^{71}$. Il s'agit en somme, pour reprendre la formule du Rousseau, de donner à voir le remède dans le $\mathrm{mal}^{72}$.

L'on peut toutefois rencontrer des états métamorphiques qui ne soient pas seulement dégradants mais qui puissent signifier une progressive réformation et domination du corps. De tels exemples se trouvent davantage dans la littérature spirituelle ou mystique et nous souhaiterions ne citer brièvement qu'un seul exemple, celui d'Ignacio de Loyola. Dans son autobiographie rédigée par Cándido de Damases, l'on relate la jeunesse tumultueuse du futur saint et son goût pour le métier des armes qui lui vaut une blessure très grave lors d'un combat contre les Français à Pampelune. Cette modification de son équilibre corporel donne lieu à un véritable exercice de transformation du corps qui passe par la maitrise de la douleur et le bouleversement de la forme de certaines parties du corps :

4. Y viniendo ya los huesos a soldarse unos con otros, le quedó abajo de la rodilla un hueso encabalgado sobre otro, por lo cual la pierna quedaba más corta; y quedaba alli el hueso tan levantado, que era cosa fea; lo cual él no pudiendo sufrir, porque determinaba seguir el mundo, y juzgaba que aquello lo afearía, se informó de los cirujanos si se podía aquello cortar; y ellos dijeron que bien se podia cortar; mas que los dolores serian mayores que todos los que habia pasado, por estar aquello ya sano, y ser menester espacio para cortarlo; y todavía él se determinó martirizarse por su propio gusto, aunque su hermano más viejo se espantaba y decía que tal dolor él no se atrevería a sofrir; lo cual el herido sufrió con la sólita paciencia.

5. Y cortada la carne y el hueso que allí sobraba, se atendió a usar de remedios para que la pierna no quedase tan corta, dándole muchas unturas, y estendiéndola con instrumentos continuamente, que muchos dias le martirizaban. Mas nuestro Señor le fue dando salud; y se fue hallando tan bueno, que en todo lo demás estaba sano, sino que no podía tenerse bien sobre la pierna, y asi le era forzado estar en el lecho ${ }^{73}$.

Le retour à l'état de santé s'est donc fait par une progressive métamorphose du corps et un dépassement de la souffrance ; un tel processus est naturellement riche de significations spirituelles et participe d'une très vaste tradition de domination du corps née avec les souffrances du Christ puis les nombreux renoncements et mortifications des premiers Pères du Désert.

71. Sur la monstruosité, voir les travaux éclairants et novateurs de Gaëlle le Gall : «Science divinatoire et observation médicale : regards croisés sur la monstruosité dans Guzmán de Alfarache de Mateo Alemán ", Actes du colloque interdisciplinaire du CELEC Figures du monstre : regards croisés dans les cultures occidentales (18-19 décembre 2008) publiés en ligne : http://cahiersducelec. univ-st-etienne.fr/ no 1, décembre 2010. L'on attend par ailleurs beaucoup de la thèse que Gaëlle le Gall prépare sous la direction du Professeur Philippe Meunier, Une poétique du corps dans les récits picaresques espagnols (1599-1610).

72. Jean Starobinski, Le remède dans le mal, Paris, Gallimard, coll. « Les Essais », 1989.

73. Ignacio de Loyola, "Autobiografía por Cándido de Damases ", Obras, Madrid, BAC, 1997, p. 102. 
Si l'ensemble des exemples que nous avons pu donner montre à l'évidence la richesse de cette thématique de la métamorphose des corps, on aura également pu considérer la diversité des interprétations morales qui peut en être livrée. Le phénomène d'allégorisation des corps et, de manière plus générale, des œuvres que suppose la littérature baroque nous conduit paradoxalement à une saturation du sens moral (qui devient le centre de l'ensemble des constructions fictives) et à une prolifération de figuras. Les Sueños de Quevedo ou le Criticón de Baltasar Gracián en sont des preuves assez éclatantes. De ce même fait, ne pourrait-on pas considérer qu'au-delà du sens moral que celles-ci portent et incarnent, se manifeste un goût marqué pour la prolifération formelle ? Quevedo ou Gracián ne sont-ils pas prisonniers des métamorphoses qu'ils créent, fascinés par l'objet inventé ? La succession d'un discours sur la grandeur et la misère de l'homme semble brouiller le message éthique initial et permettre une pluralité d'interprétations de la progression formelle qui constitue, à n'en pas douter, le jeu même de la littérature. 
